\title{
Global Exponential Stability and Periodicity of Nonautonomous Impulsive Neural Networks with Time-Varying Delays and Reaction-Diffusion Terms
}

\author{
Weiyi Hu and Kelin Li \\ School of Mathematics and Statistics, Sichuan University of Science \& Engineering, Zigong, Sichuan 643000, China \\ Correspondence should be addressed to Kelin Li; lkl@suse.edu.cn
}

Received 30 May 2021; Revised 22 July 2021; Accepted 5 August 2021; Published 16 August 2021

Academic Editor: Sigurdur F. Hafstein

Copyright (c) 2021 Weiyi Hu and Kelin Li. This is an open access article distributed under the Creative Commons Attribution License, which permits unrestricted use, distribution, and reproduction in any medium, provided the original work is properly cited.

\begin{abstract}
In this paper, we investigate the global exponential stability and periodicity of nonautonomous cellular neural networks with reaction-diffusion, impulses, and time-varying delays. By establishing a new differential inequality for nonautonomous systems, using the properties of M-matrix and inequality techniques, some new sufficient conditions for the global exponential stability of the system are obtained. Moreover, sufficient conditions for the periodic solutions of the system are obtained by using the Poincare mapping and the fixed point theory. The validity and superiority of the main results are verified by numerical examples and simulations.
\end{abstract}

\section{Introduction}

Since Chua proposed the cellular neural networks (CNNs) in the 1980s [1], the neural network models have been widely studied and applied in the fields of signal recognition, image processing, pattern classification, and so on. Indeed, all these applications rely on the dynamic behaviors of neural networks. The key to using neural networks to solve these problems is that the neural network must be globally dynamic stable; that is to say, each of its loci must converge to a unique balance. In the practical application of neural networks, time delay is inevitable because of the limited signal transmission and switching speed. As we all know, the time delay may make the system lose stability or produce nonlinear oscillation, which makes the dynamic behaviors of the system more complicated. Under the circumstances, the neural network model with time delay is introduced to better reflect the actual state. Hence, how to ensure the stability and good performance of the system is particularly important in the case of time delay. The dynamic analysis of time-delay neural networks has received considerable attention; for example, see [2-7].
In practical applications, neurons change over time and space. In order to better describe the state of neurons, a partial differential equation that considers diffusion is introduced in space. So, in 1995, Chua et al. [8] established the structure of reaction-diffusion CNNs. In $[9,10]$, the authors have investigated the global exponential stability and the global exponential robust stability of reactiondiffusion neural networks with time-varying delays, by using partial differential equations to express the diffusion term of the CNNs, some interesting results were obtained. In [11], the authors used the properties and inequality analysis of the nonsingular M-matrix to study the exponential stability of CNNs with diffusion. In [12], considering the global exponential stability under the Neumann boundary conditions, using the properties of the M-matrix and algebraic inequality techniques, some sufficient conditions for the global exponential stability of the system are obtained. In [13], under Neumann boundary conditions, several sufficient conditions based on linear matrix inequalities for the global asymptotic stability of reactordiffusion CNNs with distributed delay are given. In $[14,15]$, the Dirichlet boundary conditions of the rectangular region 
are introduced into the neural network with reactiondiffusion. In [16], under the Dirichlet boundary conditions, a diffusive-dependent Lyapunov functional related to the diffusion term is proposed to analyze the effect of the diffusion coefficient of neurons on the dynamics of the model, and the stabilization effect of the reaction-diffusion term is studied by using the extended Wirtinger inequality. In $[17,18]$, the authors considered the exponential stability of reaction-diffusion neural networks with time delays in general spatial regions and obtained some new sufficient conditions for system stability by using Poincare's inequality technique.

As we all know, the phenomenon of nonautonomy arises in a lot of real systems frequently. In particular, when a system is in a dynamic behavior for a long time, the parameters of the system often change with time. Compared with autonomous CNNs, the study of nonautonomous CNNs is equally important. In fact, many authors have explored the dynamic behavior of different types of nonautonomous CNNs with time delays and produced some interesting results by constructing some Lyapunov-Krasovskii functionals [19-26]. In addition, some functions in these methods must be differentiable, while other features require additional constraints in terms of delay. In [27], the author improved some of the earlier Halanay-type inequalities and obtained the exponential stability of CNNs and the existence of periodic solutions through these new inequalities. In [28], sufficient conditions for the global exponential stability of a special class of nonautonomous differential systems with infinite time delay are given, without proving the existence of equilibrium point, periodic solution, or almost periodic solution. In addition, during the physical simulation of the neural network, the neural network will sometimes be interrupted due to the transient disturbance, which is called the impulse effect. Impulse may cause system instability or other more complex dynamic behavior. The influence of pulses on neural networks has been considered in many literatures [29-33]. Therefore, it is of great significance to study the stability and periodicity of neural network models with dynamic impulses, time delays, and reaction-diffusion terms.

Here, we introduce a class of new nonautonomous impulsive neural networks with time-varying delays and reaction-diffusion terms as follows:

$$
\begin{aligned}
\frac{\partial z_{p}(t, x)}{\partial t}= & \sum_{k=1}^{m} \frac{\partial}{\partial x_{k}}\left(D_{p k} \frac{\partial z_{p}(t, x)}{\partial x_{k}}\right)-\beta_{p}(t) z_{p}(t, x)+\sum_{q=1}^{n} a_{p q}(t) f_{q}\left(z_{q}(t, x)\right) \\
& +\sum_{q=1}^{n} b_{p q}(t) g_{q}\left(z_{q}\left(t-\tau_{p q}(t), x\right)\right)+J_{p}(t), \quad t \neq t_{l}, p=1,2, \ldots, n, x \in \Xi, \\
z_{p}\left(t_{l}^{+}, x\right)= & I_{p l}\left(z_{p}\left(t_{l}^{-}, x\right)\right), \quad p=1,2, \ldots, n, x \in \Xi, l \in N \triangleq\{0,1,2, \ldots\}, \\
z_{p}(t, x)= & 0, \quad t \geq 0, x \in \partial \Xi, \\
z_{p}(s, x)= & \mu_{p}(s, x), \quad s \in[-\tau, 0], p=1,2, \ldots, n,
\end{aligned}
$$

where $z_{p}(t, x)$ is the state of the $p$ th neuron in space $x$ and time t. $x=\left(x_{1}, x_{2}, \ldots, x_{m}\right)^{T} \in \Xi \subset R^{m} . \Xi=\left\{x=\left(x_{1}, x_{2}\right.\right.$, $\left.\left.\ldots, x_{m}\right)^{T} \| x_{k} \mid<L_{k}, k=1,2, \ldots, m\right\}$ is a bounded compact set with smooth boundary $\partial \Xi$ and mes $\Xi>0$ in space $R^{m}\left(L_{k}\right.$ is a positive constant). $z(t, x)=\left(z_{1}(t, x), z_{2}(t, x), \ldots, z_{n}(t\right.$, $x))^{T} \in R^{n} . \beta_{p} \in C\left(R_{+}, R_{+}\right)$denotes the rate at which the $p$ th neuron resets its potential to the isolated resting state when disconnected from the network and external input; $a_{p q} \in$ $C\left(R_{+}, R\right)$ denotes the intensity of the $p$ th element on the $q$ th element at time $t ; b_{p q} \in C\left(R_{+}, R\right)$ represents the strength of the $q$ th element due to delay at the $p$ th element. $\tau_{p q}(t)$ represents the transmission delay from the $p$ th element to the $q$ th element and satisfies $0 \leq \tau_{p q}(t) \leq \tau_{p q} \leq \tau(\tau=$ $\left.\max _{1 \leq p, q \leq n}\left\{\tau_{p q}\right\}\right) ; f_{q} \in C(R, R)$ and $g_{q} \in C(R, R)$ denote the activation function and activation function caused by delay, respectively; $J_{p}(t)$ represents the external input of the $p$ th neuron at time $t$. In (1b), $t_{l}>0$ satisfies $t_{l}<t_{l+1}, \lim _{l \longrightarrow+\infty} t_{l}=$ $+\infty ; z_{p}\left(t_{l}^{-}, x\right)$ and $z_{p}\left(t_{l}^{+}, x\right)$ represent the left and right limits at $t_{k}$, respectively; $I_{p k}$ shows impulsive perturbation of the $p$ th neuron at time $t_{k}$. Let $z_{p}\left(t_{l}^{+}, x\right)=z_{p}\left(t_{l}, x\right), l \in N$. Equation (1c) denotes the Dirichlet boundary conditions and $(1 \mathrm{~d})$ denotes the initial conditions.

If impulsive operator $I_{p l}\left(z_{p}\right)=0, p=1,2, \ldots, n, l \in N$, systems (1a)-(1d) will become a model of the following form: 


$$
\begin{aligned}
\frac{\partial z_{p}(t, x)}{\partial t}= & \sum_{k=1}^{m} \frac{\partial}{\partial x_{k}}\left(D_{p k} \frac{\partial z_{p}(t, x)}{\partial x_{k}}\right)-\beta_{p}(t) z_{p}(t, x)+\sum_{q=1}^{n} a_{p q}(t) f_{q}\left(z_{q}(t, x)\right) \\
& +\sum_{q=1}^{n} b_{p q}(t) g_{q}\left(z_{q}\left(t-\tau_{p q}(t), x\right)\right)+J_{p}(t), \quad p=1,2, \ldots, n, x \in \Xi, \\
z_{p}(t, x)= & 0, \quad t \geq 0, x \in \partial \Xi \\
z_{p}(s, x)= & \mu_{p}(s, x), \quad s \in[-\tau, 0], p=1,2, \ldots, n .
\end{aligned}
$$

Systems (2a)-(2c) are called the continuous form of impulsive systems (1a)-(1d).

The main contributions of this work are as follows:

(I) We have formulated a class of new neural network models which assembles nonautonomous neural networks, reaction-diffusion cellular neural networks with time-varying delays, impulses, and the Dirichlet boundary conditions.

(II) We have obtained several new criteria which guarantee the global exponential stability and periodicity of systems (1a)-(1d). These criteria are shown in simple algebraic inequality forms, and they rely only on the system parameters.

(III) An optimization method is proposed in order to estimate the exponential convergence rate of systems (1a)-(1d).

The remainder of this paper is organized as follows. In Section 2, some assumptions and the necessary knowledge are provided. We give our main results in Section 3. In Section 4, two examples are given to show the effectiveness of the results obtained here. Finally, we give the conclusion in Section 5.

\section{Preliminaries}

In this section, we make some assumptions and introduce some notions.

(A1) For the activation functions $f_{p}(z)$ and $g_{p}(z)$, there exist positive diagonal matrices $F=$ diag $\left(F_{1}, F_{2}, \ldots, F_{n}\right)$ and $G=\operatorname{diag}\left(G_{1}, G_{2}, \ldots, G_{n}\right)$ such that

$$
\begin{gathered}
F_{p}=\sup _{z \neq w}\left|\frac{f_{p}(z)-f_{p}(w)}{z-w}\right|, \\
G_{p}=\sup _{z \neq w}\left|\frac{g_{p}(z)-g_{p}(w)}{z-w}\right|,
\end{gathered}
$$

for all $z, w \in R(z \neq w), p=1,2, \ldots, n$.

(A2) There exists a positive diagonal matrix $\mathfrak{\Im}_{l}=\operatorname{diag}\left(I_{1 l}, \ldots, I_{n l}\right)$ such that

$$
\left|I_{p l}(z)-I_{p l}(z)\right| \leq I_{p l}|z-v|
$$

for all $z, v \in R(z \neq v), p \in\{1,2, \ldots, n\}, l \in N$.

(A3) $a_{p q}(t), b_{p q}(t), \beta_{p}(t), J_{p}(t)$, and $\tau_{p q}(t)$ are continuous bounded functions defined on $t \in[0,+\infty)$ for all $p, q \in\{1,2, \ldots, n\}$.

We define $\left\|z_{p}(t, x)\right\|_{2}=\left[\int_{\Xi}\left|z_{p}(t, x)\right|^{2} \mathrm{~d} x\right]^{(1 / 2)}$, $p=1,2, \ldots, n . \quad R_{+}=[0,+\infty)$ and $h^{+}(t)=\max \{0, h(t)\}$, where $h(t)$ is a continuous function. For any $\omega(s, x)=\left(\omega_{1}(s, x), \omega_{2}(s, x), \ldots, \omega_{n}(s, x)\right)^{T} \in P C(\Xi)$, the norm on $P C(\Xi)$ is defined by

$$
\|\omega\|=\sup _{-\tau \leq s \leq 0} \sum_{p=1}^{n}\left\|\omega_{p}(s, x)\right\|_{2} .
$$

$P C(\Xi) \triangleq\left\{\omega:[-\tau, 0] \times \Xi \longrightarrow R^{n} \mid \omega(s, x)\right.$ is bounded on $[-\tau, 0] \times \Xi$ and $\omega\left(s^{+}, x\right)=\omega(s, x)$ for $s \in[-\tau, 0] . \omega\left(s^{-}, x\right)$ exists for $s \in[-\tau, 0]$ and $\omega\left(s^{-}, x\right)=\omega(s, x)$ for all but a finite number of points $s \in[-\tau, 0]\}$.

$P C \triangleq\left\{\omega:[-\tau, 0] \longrightarrow R^{n} \mid \omega(s)\right.$ is bounded on $[-\tau, 0]$ and $\omega\left(s^{+}\right)=\omega(s)$ for $s \in[-\tau, 0] . \omega\left(s^{-}\right)$exists for $s \in[-\tau, 0]$ and $\omega\left(s^{-}\right)=\omega(s)$ for all but a finite number of points $s \in[-\tau, 0]\}$.

For $C, D \in R^{m \times n}$, we define the Schur product by $C \otimes D=\left(c_{p q} d_{p q}\right)_{m \times n}$, and $C \geq D(C>D)$ means that the inequality $c_{p q} \geq b_{p q}\left(c_{p q}>d_{p q}\right)$ holds. $\mathbf{e}=(1,1, \ldots, 1)^{T} \in R^{n}$ and $E$ is a $n$-dimensional identity matrix.

Definition 1. A function $z(t, x)\left(z:[-\tau,+\infty) \times \Xi \longrightarrow R^{n}\right)$ is said to be the solution of system (1a)-(1d) if $z(t, x)$ satisfies the following conditions:

(1) For $t, z(t, x)$ is piecewise continuous with the first kind of discontinuity at the points $t_{l}, l \in N$, and $z(t, x)$ is right-continuous at every discontinuity point

(2) $z(t, x)$ satisfies (1a)-(1c) for all $t \geq 0$, and $z(t, x)=$ $\mu(t, x)$ for $t \in[-\tau, 0]$

Therefore, $z(t, x)$ is a particular solution that satisfies the initial conditions for systems (1a)-(1d), and $z(t, \mu, x)$ represents the special solution of systems (1a)-(1d) under initial condition $\mu \in P C(\Xi)$.

Definition 2. $z(t, \omega, x)$ is a particular solution of systems (1a)-(1d) and satisfies the initial conditions $\omega \in P C(\Xi)$. In the initial condition of $\omega \in P C(\Xi), z(t, \omega, x)$ is any solution 
to systems (1a)-(1d). If there is a positive number $\lambda>0$ and $M \geq 1$ such that

$$
\|z(t, \omega, x)-z(t, \oplus, x)\| \leq M\|\omega-\oplus\| e^{-\lambda t}, \quad \text { for all } t \geq 0,
$$

then systems (1a)-(1d) are said to be globally exponentially stable.

Definition 3 (see [34]). A real matrix $D=\left(d_{p q}\right)_{n \times n}$ is said to be a nonsingular $M$-matrix if $d_{p q} \leq 0, p, q=1,2, \ldots, n, p \neq q$, and all successive principal minors of $D$ are positive.

Lemma 1 (see [34]). Let $D=\left(d_{p q}\right)_{n \times n}$ with $d_{p q} \leq 0(p \neq q)$, then $D$ is a nonsingular M-matrix if and only if the diagonal elements of $D$ are all positive, and there exists a positive vector $d$ such that $D d>0$ or $D^{T} d>0$.

Lemma 2 (see [35]). Let $\Xi$ be a cube $\left|x_{k}\right|<L_{k}(k=1,2, \ldots$, $m)$, and let $h(x)$ be a real-valued function belonging to $C^{1}(\Xi)$ which vanishes on the boundary an of $\Xi$, i.e., $\left.h(x)\right|_{\partial \Xi}=0$. Then,

$$
\int_{\Xi} h^{2}(x) \mathrm{d} x \leq L_{k}^{2} \int_{\Xi}\left|\frac{\partial h}{\partial x_{k}}\right|^{2} \mathrm{~d} x
$$

Lemma 3. If $a(x)$ and $b(x)$ are continuous on $\left[x_{0},+\infty\right)$ with $a(x)-b(x) \geq \rho>0$ for all $x \in\left[x_{0},+\infty\right)$ and there is a positive number $L$ such that $0 \leq b(x) \leq L$ for all $x \geq x_{0}$, then $\lambda^{*} \equiv \inf _{x \geq x_{0}}\left\{\lambda>0: \lambda-a(x)+b(x) e^{\lambda \tau(x)}=0\right\}>0$, where $\tau(x)$ is nonnegative function (i.e., $\tau(x) \geq 0$ for all $x \geq 0$ ).

Proof. Set $F(\lambda)=\lambda-a(x)+b(x) \mathrm{e}^{\lambda \tau(x)}$. Note that

$$
\begin{aligned}
F(0) & =-a(x)+b(x)<0, \\
\frac{\mathrm{d} F}{\mathrm{~d} \lambda} & =1+\tau(x) b(x) \mathrm{e}^{\lambda \tau(x)}>0 \text { and } F(+\infty)>0,
\end{aligned}
$$

then we know that $F(\lambda)$ is a strictly monotone increasing function. Hence, for any $x \geq x_{0}$, there exists a unique positive $\lambda(x)$ such that

$$
\lambda(x)-a(x)+b(x) \mathrm{e}^{\lambda(x) \tau(x)}=0
$$

Therefore, $\lambda^{*}$ exists and $\lambda^{*} \geq 0$. We will prove $\lambda^{*}>0$ in the following.

When $\tau(x)=0$, we can obtain that $\lambda^{*}=a(x)-b(x) \geq \rho>0$.

When $\quad \tau(x)>0, \quad \lambda^{*}<0$. Here, for any $\sigma<\min \{(\rho / 2),(1 / \tau(x)) n(1+(\rho / 2 L))\}$, there exists $x^{*}>0$ such that $\lambda^{*}\left(x^{*}\right)<\sigma$ and satisfies

$$
\lambda^{*}\left(x^{*}\right)-a\left(x^{*}\right)+b\left(x^{*}\right) e^{\lambda^{*}\left(x^{*}\right) \tau\left(x^{*}\right)}=0 .
$$

It follows that

$$
\begin{aligned}
0= & \lambda^{*}\left(x^{*}\right)-a\left(x^{*}\right)+b\left(x^{*}\right) e^{\lambda^{*}\left(x^{*}\right) \tau\left(x^{*}\right)} \\
& <\sigma-a\left(x^{*}\right)+b\left(x^{*}\right)\left(1+\frac{\rho}{2 L}\right) \\
& <\frac{\rho}{2}-\left(a\left(x^{*}\right)-b\left(x^{*}\right)\right)+b\left(x^{*}\right) \frac{\rho}{2 L} \\
& <\frac{\rho}{2}-\rho+\frac{\rho}{2}=0,
\end{aligned}
$$

and this is a contradiction. So, $\lambda^{*}>0$. The proof is completed.

Remark 1. Lemma 3 is a generalization of Lemma 4.1 in [36].

Lemma 4. Let $\tau>0, a<b \leq+\infty$, suppose that $Z(t)=\left(Z_{1}\right.$ $\left.(t), Z_{2}(t), \ldots, Z_{n}(t)\right)^{T} \in C\left[[a, b), R^{n}\right]$ satisfies the following differential inequality:

$$
\begin{cases}D^{+} Z(t) \leq P(t) Z(t)+(Q(t) \otimes \bar{Z}(t)) \mathbf{e}, & a \leq t<b, \\ Z(a+s) \in P C, & -\tau \leq s<0\end{cases}
$$

where $P(t)=\left(p_{p q}(t)\right)_{n \times n}$ with $p_{p q}(t) \geq 0(p \neq q), Q(t)$ $=\left(q_{p q}(t)\right)_{n \times n}$ with $q_{p q}(t) \geq 0, \bar{Z}(t)=\left(Z\left(t-\tau_{p q}(t)\right)\right)_{n \times n}$. If the initial condition satisfies

$$
Z(t) \leq \kappa \xi e^{-\lambda(t)}, \quad \kappa \geq 0, t \in[a-\tau, a]
$$

in which the scalar $\lambda>0$ and $\xi=\left(\xi_{1}, \xi_{2}, \ldots, \xi_{n}\right)^{T}>0$ are determined by

$$
[\lambda E+P(t)+Q(t) \otimes \zeta(\lambda)] \xi<0, \quad \zeta(\lambda)=\left(e^{\lambda \tau_{p q}(t)}\right)_{n \times n}
$$

then $Z(t) \leq \kappa \xi e^{-\lambda(t-a)}$, for $t \in[a, b)$.

Proof. For $p \in\{1,2, \ldots, n\}$ and arbitrary $\varepsilon>0$, set $w_{p}(t) \triangleq(\kappa+\varepsilon) \xi_{p} e^{-\lambda(t-a)}$. We prove that

$$
Z_{p}(t) \leq w_{p}(t)=(\kappa+\varepsilon) \xi_{p} e^{-\lambda(t-a)}, \quad t \in[a, b), p=1,2, \ldots, n .
$$

If this is not true, then there exist a number $t^{*} \in[a, b)$ and some integer $r$ such that

$$
\begin{aligned}
Z_{r}\left(t^{*}\right) & =w_{r}\left(t^{*}\right), \\
D^{+} Z_{r}\left(t^{*}\right) & \geq \dot{w}_{r}\left(t^{*}\right), \\
Z_{p}(t) & \leq w_{p}(t), \quad t \in\left[a, t^{*}\right], p=1,2, \ldots, n .
\end{aligned}
$$

From Lemma 4 and (15), we have 


$$
\begin{aligned}
D^{+} Z_{r}\left(t^{*}\right) & \leq \sum_{q=1}^{n}\left[p_{r q}\left(t^{*}\right) Z_{q}\left(t^{*}\right)+q_{r q}\left(t^{*}\right) Z_{q}\left(t^{*}-\tau_{r q}\left(t^{*}\right)\right)\right] \\
& \leq \sum_{q=1}^{n}\left[p_{r q}\left(t^{*}\right)(\kappa+\varepsilon) \xi_{q} e^{-\lambda\left(t^{*}-a\right)}+q_{r q}\left(t^{*}\right)(\kappa+\varepsilon) \xi_{q} e^{-\lambda\left(t^{*}-\tau_{r q}\left(t^{*}\right)-a\right)}\right] \\
& =\sum_{q=1}^{n}\left[p_{r q}\left(t^{*}\right)(\kappa+\varepsilon) \xi_{q} e^{-\lambda\left(t^{*}-a\right)}+q_{r q}\left(t^{*}\right) e^{\lambda \tau_{r q}\left(t^{*}\right)}(\kappa+\varepsilon) \xi_{q} e^{-\lambda\left(t^{*}-a\right)}\right] \\
& \leq \sum_{q=1}^{n}\left[p_{r q}\left(t^{*}\right)+q_{r q}\left(t^{*}\right) e^{\lambda \tau_{r q}\left(t^{*}\right)}\right](\kappa+\varepsilon) \xi_{q} e^{-\lambda\left(t^{*}-a\right)} .
\end{aligned}
$$

According to $[\lambda E+P(t)+Q(t) \otimes \zeta(\lambda)] \xi<0, p_{p q}\left(t^{*}\right)$ $\geq 0(p \neq q), q_{p q}\left(t^{*}\right) \geq 0$, and $e^{\lambda \tau p q}\left(t^{*}\right)>0$, it follows that

$$
\sum_{q=1}^{n}\left[p_{r q}\left(t^{*}\right)+q_{r q}\left(t^{*}\right) e^{\lambda \tau_{r q}\left(t^{*}\right)}\right] \xi_{q}<-\lambda \xi_{r}<0 .
$$

From (17), we have

$$
D^{+} Z_{r}\left(t^{*}\right)<-\lambda \xi_{r}(\kappa+\varepsilon) e^{-\lambda\left(t^{*}-l\right)}=\dot{w}_{r}\left(t^{*}\right),
$$

that is,

$$
D^{+} Z_{r}\left(t^{*}\right)<\dot{w}_{r}\left(t^{*}\right) .
$$

It is clear that this inequality and (16) contradict each other, and this implies that inequality (15) holds for all $t \in[a, b)$.

Letting $\varepsilon \longrightarrow 0$, we obtain that

$$
Z_{p}(t) \leq \kappa \xi_{p} e^{-\lambda(t-a)}, \quad p=1,2, \ldots, n, t \in[a, b) .
$$

The proof is completed.

\section{Main Results}

In this part, we establish a series of sufficient conditions to ensure the global exponential stability of systems (1a)-(1d).

Theorem 1. Under assumptions (A1)-(A3), suppose that

(C1) There exist a vector $\xi=\left(\xi_{1}, \xi_{2}, \ldots, \xi_{n}\right)^{T}>0$ and $a$ constant $\lambda>0$ such that

$$
[\lambda E-D(t)+A(t) F+B(t) G \otimes \zeta(\lambda)] \xi<0, \quad t \geq 0,
$$

where $D(t)=\operatorname{diag}\left(d_{1}(t), d_{2}(t), \ldots, d_{n}(t)\right)$ with $d_{p}(t)$ $=\beta_{p}(t)+\sum_{k=1}^{m}\left(D_{p k} / L_{k}^{2}\right), \quad \zeta(\lambda)=\left(e^{\lambda \tau_{p q}(t)}\right)_{n \times n}, \quad A(t)=$ $\left(a_{p q}^{+}(t)\right)_{n \times n}, B(t)=\left(b_{p q}^{+}(t)\right)_{n \times n}, \quad F=\operatorname{diag}\left(F_{1}, F_{2}, \ldots\right.$, $\left.F_{n}\right)$, and $G=\operatorname{diag}\left(G_{1}, G_{2}, \ldots, G_{n}\right)$;

(C2) There is a positive number $\gamma>0$ such that

$$
\sup _{l \in N}\left\{\frac{\ln \gamma_{l}}{t_{l}-t_{l-1}}\right\} \leq \gamma<\lambda,
$$

where $\gamma_{l}=\max _{1 \leq p \leq n}\left\{1, I_{p l}\right\}, l \in N$; then, systems (1a)-(1d) are globally exponentially stable.

Proof. For $\omega, \varpi \in P C(\Xi)$, we denote, respectively, the solutions of systems $(1 \mathrm{a})-(1 \mathrm{~d})$ through $(0, \omega)$ and $(0, \infty)$ :

$$
\begin{aligned}
& z(t, \omega, x)=\left(z_{1}(t, \omega, x), z_{2}(t, \omega, x), \ldots, z_{n}(t, \omega, x)\right)^{T}, \\
& z(t, \omega, x)=\left(z_{1}(t, \omega, x), z_{2}(t, \omega, x), \ldots, z_{n}(t, \omega, x)\right)^{T} .
\end{aligned}
$$

Define $\quad z_{t}(t, \omega, x)=z(t+s, \omega, x), z_{t}(\Phi, x)=z(t+s$, $\Phi, x),-\tau<s \leq 0, t \geq 0$, then $z_{t}(\omega, x), z_{t}(\omega, x) \in P C(\Xi)$ for $t \geq 0$.

Let $Z_{p}(t, \psi, x)=z_{p}(t, \omega, x)-z_{p}(t, \omega, x), p=1,2, \ldots$, $n, \psi=\omega-\emptyset$, we have

$$
\begin{aligned}
\frac{\partial Z_{p}(t, \psi, x)}{\partial t}= & \sum_{k=1}^{m} \frac{\partial}{\partial x_{k}}\left(D_{p k} \frac{\partial Z_{p}(t, \psi, x)}{\partial x_{k}}\right)-\beta_{p}(t) Z_{p}(t, \psi, x) \\
& -\sum_{q=1}^{n} a_{p q}(t)\left[f_{q}\left(z_{q}(t, \omega, x)\right)-f_{q}\left(z_{q}(t, \omega, x)\right)\right] \\
& -\sum_{q=1}^{n} b_{p q}(t)\left[g_{q}\left(z_{q}\left(t-\tau_{p q}(t), \omega, x\right)\right)-g_{q}\left(z_{q}\left(t-\tau_{p q}(t)\right), \Phi, x\right)\right],
\end{aligned}
$$




$$
\begin{aligned}
& \frac{\mathrm{d}}{\mathrm{d} t} \int_{\Xi}\left(Z_{p}(t, \psi, x)\right)^{2} \mathrm{~d} x \\
& =2 \int_{\Xi} Z_{p}(t, \psi, x) \sum_{k=1}^{m} \frac{\partial}{\partial x_{k}}\left(D_{p k} \frac{\partial Z_{p}(t, \psi, x)}{\partial x_{k}}\right) \mathrm{d} x-2 \int_{\Xi} \beta_{p}(t) Z_{p}^{2}(t, \psi, x) \mathrm{d} x \\
& \quad+2 \sum_{q=1}^{n} a_{p q}(t) \int_{\Xi} Z_{p}(t, \psi, x)\left[f_{q}\left(z_{q}(t, \omega, x)\right)-f_{q}\left(z_{q}(t, \omega, x)\right)\right] \mathrm{d} x \\
& \quad+2 \sum_{q=1}^{n} b_{p q}(t) \int_{\Xi} Z_{p}(t, \psi, x)\left[g_{q}\left(z_{q}\left(t-\tau_{p q}(t), \omega, x\right)\right)-g_{q}\left(z_{q}\left(t-\tau_{p q}(t)\right), \omega, x\right)\right] \mathrm{d} x .
\end{aligned}
$$

From the Dirichlet boundary conditions and Greens formula, we can obtain

By Lemma 2, we have

$$
\begin{gathered}
\int_{\Xi} Z_{p}(t, \psi, x) \sum_{k=1}^{m} \frac{\partial}{\partial x_{k}}\left(D_{p k} \frac{\partial Z_{p}(t, \psi, x)}{\partial x_{k}}\right) \mathrm{d} x \\
=-\sum_{k=1}^{m} \int_{\Xi} D_{p k}\left(\frac{\partial\left(Z_{p}(t, \psi, x)\right)}{\partial x_{k}}\right)^{2} \mathrm{~d} x .
\end{gathered}
$$

$\int_{\Xi} Z_{p}(t, \psi, x) \sum_{k=1}^{m} \frac{\partial}{\partial x_{k}}\left(D_{p k} \frac{\partial Z_{p}(t, \psi, x)}{\partial x_{k}}\right) \mathrm{d} x \leq-\sum_{k=1}^{m} \frac{D_{p k}}{L_{k}^{2}} \int_{\Xi}\left(Z_{p}(t, \psi, x)\right)^{2} \mathrm{~d} x=-\sum_{k=1}^{m} \frac{D_{p k}}{L_{k}^{2}}\left\|Z_{p}(t, \psi, x)\right\|_{2}^{2}$.

From (A1)-(A3) and Hoder inequality, we can get In a similar way, we have

$$
\begin{aligned}
& \sum_{q=1}^{n} a_{p q}(t) \int_{\Xi} Z_{p}(t, \psi, x)\left[f_{q}\left(z_{q}(t, \omega, x)\right)-f_{q}\left(z_{q}(t, \omega, x)\right)\right] \mathrm{d} x \\
& \quad \leq \sum_{q=1}^{n} a_{p q}^{+}(t) \int_{\Xi}\left|Z_{p}(t, \psi, x) \| f_{q}\left(z_{q}(t, \omega, x)\right)-f_{q}\left(z_{q}(t, \omega, x)\right)\right| \mathrm{d} x \\
& \quad \leq \sum_{q=1}^{n} a_{p q}^{+}(t) \int_{\Xi}\left|Z_{p}(t, \psi, x) \| Z_{q}(t, \psi, x)\right| F_{q} \mathrm{~d} x \\
& \quad \leq \sum_{q=1}^{n} a_{p q}^{+}(t)\left\|Z_{p}(t, \psi, x)\right\|_{2} F_{q}\left\|Z_{q}(t, \psi, x)\right\|_{2} .
\end{aligned}
$$

$$
\begin{aligned}
& \sum_{q=1}^{n} b_{p q}(t) \int_{\Xi} Z_{p}(t, \psi, x)\left[g_{q}\left(z_{q}\left(t-\tau_{p q}(t), \omega, x\right)\right)-g_{q}\left(z_{q}\left(t-\tau_{p q}(t)\right), \Phi, x\right)\right] \mathrm{d} x \\
& \quad \leq \sum_{q=1}^{n} b_{p q}^{+}(t)\left\|Z_{q}\left(t-\tau_{p q}(t), \psi, x\right)\right\|_{2} G_{q}\left\|Z_{p}(t, \psi, x)\right\|_{2} .
\end{aligned}
$$


Applying (26)-(30) to (25), we can obtain

$$
\begin{aligned}
\frac{1}{2} \frac{\mathrm{d}}{\mathrm{d} t}\left\|Z_{p}(t, \psi, x)\right\|_{2}^{2} \leq & -\left(\beta_{p}(t)+\sum_{k=1}^{m} \frac{D_{p k}}{L_{k}^{2}}\right)\left\|Z_{p}(t, \psi, x)\right\|_{2}^{2}+\sum_{q=1}^{n} a_{p q}^{+}(t)\left\|Z_{p}(t, \psi, x)\right\|_{2} F_{q}\left\|Z_{p}(t, \psi, x)\right\|_{2} \\
& +\sum_{q=1}^{n} b_{p q}^{+}(t)\left\|Z_{q}\left(t-\tau_{p q}(t), \psi, x\right)\right\|_{2} G_{q}\left\|Z_{p}(t, \psi, x)\right\|_{2},
\end{aligned}
$$

i.e.,

$$
D^{+}\left\|Z_{p}(t, \psi, x)\right\|_{2} \leq-\left(\beta_{p}(t)+\sum_{k=1}^{m} \frac{D_{p k}}{L_{k}^{2}}\right)\left\|Z_{p}(t, \psi, x)\right\|_{2}+\sum_{q=1}^{n} a_{p q}^{+}(t) F_{q}\left\|Z_{q}(t, \vartheta, x)\right\|_{2}+\sum_{q=1}^{n} b_{p q}^{+}(t)\left\|Z_{q}\left(t-\tau_{p q}(t), \psi, x\right)\right\|_{2} G_{q} .
$$

Let $\quad Z_{p}(t)=\left\|Z_{p}(t, \psi, x)\right\|_{2}, Z(t)=\left(Z_{1}(t), Z_{2}(t), \ldots\right.$, $\left.Z_{n}(t)\right)^{T}, \bar{Z}(t)=\left(Z_{p}\left(t-\tau_{p q}(t)\right)\right)_{n \times n}, d_{p}(t)=\beta_{p}(t)+\sum_{k=1}^{m}$ $\left(D_{p k} / L_{k}^{2}\right), p=1,2, \ldots, n, D(t)=\operatorname{diag}\left(d_{1}(t), d_{2}(t), \ldots, d_{n}\right.$ $(t))$, and $P(t)=-D(t)+A(t) F, Q(t)=B(t) G$. Then, (31) can be reduced to the following form:

$$
D^{+} Z(t) \leq P(t) Z(t)+(Q(t) \otimes \bar{Z}(t)) \mathbf{e} .
$$

From condition (C1), there exist a vector $\xi=\left(\xi_{1}, \xi_{2}, \ldots, \xi_{n}\right)^{T}>0$ and a constant $\lambda>0$ such that

$$
[\lambda E-D(t)+A(t) F+B(t) G \otimes \zeta(\lambda)] \xi<0 .
$$

Taking $\kappa=\|\psi\| / \min _{1 \leq p \leq n}\left\{\xi_{p}\right\}$, obviously

$$
Z(t) \leq \kappa \xi e^{-\lambda t}, \quad t \in\left[-\tau, t_{0}\right], t_{0}=0 .
$$

By Lemma 4, we have

$$
Z(t) \leq \kappa \xi e^{-\lambda t}, \quad t \in\left[t_{0}, t_{1}\right) .
$$

Assume that the following inequality holds for $m \leq l$ :

$Z(t) \leq \kappa \gamma_{0}, \ldots, \gamma_{m-1} \xi e^{-\lambda t}, \quad t \in\left[t_{m-1}, t_{m}\right), \gamma_{0}=1$.

When $m=l+1$, we can obtain

$$
\begin{aligned}
Z\left(t_{l}\right) & =\left\|z\left(t_{l}, \omega, x\right)-z\left(t_{l}, \Phi, x\right)\right\|_{2} \\
& =\left\|I_{l}\left(z\left(t_{l}^{-}, \omega, x\right)\right)-I_{l}\left(z\left(t_{l}^{-}, \Phi, x\right)\right)\right\|_{2} \\
& \leq \Im_{l}\left\|Z\left(t_{l}^{-}, \psi, x\right)\right\|_{2} \\
& =\Im_{l} Z\left(t_{l}^{-}\right) \\
& \leq \kappa \gamma_{0}, \ldots, \gamma_{l-1} \Im_{l} \xi \lim _{t \longrightarrow t_{l}^{-}} e^{-\lambda t_{l}} \\
& \leq \kappa \gamma_{0}, \ldots, \gamma_{l-1} \gamma_{l} \xi e^{-\lambda t_{l}} .
\end{aligned}
$$

By (37) and (38) and $\gamma_{l} \geq 1$, we obtain

$$
Z(t) \leq \kappa \gamma_{0}, \ldots, \gamma_{l-1} \gamma_{l} \xi \mathrm{e}^{-\lambda t}, \quad t_{l}-\tau \leq t \leq t_{l} .
$$

Combining (34), (35), and (39) and Lemma 4, we get

$$
Z(t) \leq \kappa \gamma_{0}, \ldots, \gamma_{l-1} \gamma_{l} \xi e^{-\lambda t}, \quad t_{l} \leq t<t_{l+1} .
$$

Applying mathematical induction, we have

$$
Z(t) \leq \kappa \gamma_{0}, \ldots, \gamma_{l-1} \xi e^{-\lambda t}, \quad t_{l-1} \leq t<t_{l}, l \in N .
$$

From (C2) and (41), we obtain

$$
Z(t) \leq \kappa e^{\gamma t_{1}} e^{\gamma\left(t_{2}-t_{1}\right)}, \ldots, e^{\gamma\left(t_{l-1}-t_{l-2}\right)} \xi e^{-\lambda t} \leq \kappa \xi e^{\gamma t} e^{-\lambda t}=\kappa \xi e^{-(\lambda-\gamma) t},
$$

for all $l \in N, t_{l-1} \leq t<t_{l}$.

This implies that

$$
\begin{aligned}
\|z(t, \omega, x)-z(t, \Phi, x)\| & =\sum_{p=1}^{n}\left\|z_{p}(t, \omega, x)-z_{p}(t, \Phi, x)\right\|_{2} \\
& =\sum_{p=1}^{n} Z_{p}(t) \\
& \leq \sum_{p=1}^{n} \kappa \xi_{p} e^{-(\lambda-\gamma) t} \\
& =\frac{\sum_{p=1}^{n} \xi_{p}}{\min _{1 \leq p \leq n}\left\{\xi_{p}\right\}}\|\omega-\Phi\| e^{-(\lambda-\gamma) t},
\end{aligned}
$$

for all $t \geq 0$.

That is,

$$
\left\|z_{t}(\omega, x)-z_{t}(\varpi, x)\right\| \leq M\|\omega-\Phi\| e^{-(\lambda-\gamma) t}, \quad t \geq 0,
$$

where $\quad M=\left(\sum_{p=1}^{n} \xi_{p} / \min _{1 \leq p \leq n}\left\{\xi_{p}\right\}\right)$. The proof is completed. 
Remark 2. In Theorem 1, the condition (C2) $\left(\gamma=\sup _{l \in N}\left\{\ln \gamma_{l} /\left(t_{l}-t_{l-1}\right)\right\}\right)$ describes the influence of the impulsive intensity and the impulsive interval on the global exponential stability of systems (1a)-(1d). In the absence of impulses, the following optimization problem can be solved in order to estimate the exponential convergence rate of systems (1a)-(1d):

$$
\text { (OP) } \begin{cases}\max & \lambda \\ \text { s.t. } & (C 1) \text { holds. }\end{cases}
$$

Obviously, $\lambda$ is associated with all time delays, the diffusion coefficients, the Dirichlet boundary conditions, and system parameters. When $\gamma \in[0, \lambda)$, Theorem 1 shows that systems (1a)-(1d) are globally exponentially stable and its exponential convergence rate equals $\lambda-\gamma$. About the exponential convergence rate $\lambda$, we have the following result.

Theorem 2. If condition (C1) holds, then $\max \lambda=\lambda^{*}$, where

$$
\lambda^{*}=\inf _{t \geq 0}\{\lambda(t)>0:[\lambda(t) E-D(t)+A(t) F+B(t) G \otimes \zeta(t)] \xi=0\}>0, \quad \zeta(t)=\left(e^{\lambda(t) \tau_{p q}(t)}\right)_{n \times n} .
$$

Proof. By Lemma 3, $\lambda^{*}$ exists and $\lambda^{*}>0$. Let $\lambda=\lambda^{*}-\varepsilon$ for all $\varepsilon \in\left(0, \lambda^{*}\right)$, then

$$
\left[\left(\lambda^{*}-\varepsilon\right) E-D(t)+A(t) F+B(t) G \otimes \zeta\left(\lambda^{*}-\varepsilon\right)\right] \xi<0,
$$

for all $t \geq 0$. That is,

$$
[\lambda E-D(t)+A(t) F+B(t) G \otimes \zeta(t)] \xi<0,
$$

for all $t \geq 0$. Therefore, $\max \lambda=\lambda^{*}$. The proof is completed.

Remark 3. Some existing neural network models become special cases of systems (2a)-(2c) formulated in this paper; for example, see $[15,16]$. It is not hard to find out that all criteria in [15] are improved. Comparing with LMIs method in [16], our results are more concise, and these results do require the differentiability on time-varying delays, so our method has different advantages and disadvantages with the method in [16]. In addition, although those models in $[37,38]$ are different from systems $(2 a)-(2 c)$ because of the difference in reaction-diffusion terms and boundary conditions, all criteria in [37] are independent of diffusion effect. In [38], the authors had, respectively, obtained global exponential stability criteria of nonautonomous neural networks with time-varying delays and reaction-diffusion terms under the Neumann boundary conditions and the Dirichlet boundary conditions. However, our approach is similar to the one in [38] when the Dirichlet boundary conditions are considered.

Remark 4. Let $\Delta(t)=D(t)-A(t) F-B(t) G$, then condition (C1) is equivalent to that $\Delta(t)$ is a nonsingular M-matrix for any $t \geq 0$. In fact, if $\Delta(t)$ is a nonsingular M-matrix for any $t \geq 0$, from Lemma 1 , there exists a vector $\xi=\left(\xi_{1}, \xi_{2}, \ldots, \xi_{n}\right)^{T}>0$ such that

$$
[D(t)-A(t) F-B(t) G] \xi>0 .
$$

Because of the uniform continuity, there exists a real number $\lambda>0$ such that

$$
[\lambda E-D(t)+A(t) F+B(t) G \otimes \theta(\lambda)] \xi<0,
$$

and this shows that condition (C1) holds. Reversely, let $\lambda=0$ in (C1); it is easy to obtain that $\Delta(t)$ is a nonsingular M-matrix for $t \geq 0$.

Based on the above discussion, we have the following conclusions.

Corollary 1. Under assumptions $(A 1)-(A 3)$, suppose that $\left(C 1^{\prime}\right)$ There exist some numbers $\xi_{p}>0(p=1,2, \ldots, n)$ and a number $\lambda>0$ such that

$$
\left(\beta_{p}(t)+\sum_{k=1}^{m} \frac{D_{p k}}{L_{k}^{2}}\right)-\sum_{q=1}^{n} \frac{\xi_{q}}{\xi_{p}} a_{p q}^{+}(t) F_{q}-\sum_{q=1}^{n} \frac{\xi_{q}}{\xi_{p}} b_{p q}^{+}(t) e^{\lambda \tau_{p q}(t)} G_{q}>\lambda>0, \quad \text { for all } t \geq 0, p=1,2, \ldots, n \text {. }
$$

(C2) There is a positive number $\gamma>0$ such that

$$
\sup _{l \in N}\left\{\frac{\ln \gamma_{l}}{t_{l}-t_{l-1}}\right\} \leq \gamma<\lambda
$$

where $\gamma_{l}=\max _{1 \leq p \leq n}\left\{1, I_{p l}\right\}, l \in N$; then, systems (1a)-(1d) are globally exponentially stable.

Proof. Note that $(\mathrm{C} 1)$ and $\left(\mathrm{Cl}^{\prime}\right)$ are equivalent; it follows from Theorem 1 that Corollary 1 holds. 
Corollary 2. Under assumptions $(A 1)$ and $(A 3)$, if condition (C1) holds, then systems (2a)-(2c) are globally exponentially stable.
Corollary 3. Under assumptions (A1) and (A3), if there exist some numbers $\xi_{p}>0(p=1,2, \ldots, n)$ and a number $\lambda>0$ such that

$$
\xi_{p}\left(\beta_{p}(t)+\sum_{k=1}^{m} \frac{D_{p k}}{L_{k}^{2}}\right)-\sum_{q=1}^{n} \xi_{q} a_{p q}^{+}(t) F_{q}-\sum_{q=1}^{n} \xi_{q} b_{p q}^{+}(t) G_{q}>\lambda>0, \quad \text { for all } t \geq 0, p=1,2, \ldots, n
$$

then systems (2a)-(2c) are globally exponentially stable.

Corollary 4. Under assumptions $(A 1)-(A 3)$, suppose that
$\left(C 1^{\prime \prime}\right)$ There exist a vector $\xi=\left(\xi_{1}, \xi_{2}, \ldots, \xi_{n}\right)^{T}>0$ and $a$ positive number $\lambda>0$ such that

$$
[\lambda E-D(t)+A(t) F+B(t) G \otimes \bar{\zeta}(\lambda)] \xi<0, \quad \bar{\zeta}(\lambda)=\left(e^{\lambda \tau}\right)_{n \times n}, t \geq 0
$$

where $D(t)=\operatorname{diag}\left(d_{1}(t) d_{2}(t), \ldots, d_{n}(t)\right)$ with $d_{p}(t)=$ $\beta_{p}(t)+\sum_{k=1}^{m}\left(D_{p k} / L_{k}^{2}\right), A(t)=\left(a_{p q}^{+}(t)\right)_{n \times n}, B(t)=\left(b_{p q}^{+}\right.$ $(t))_{n \times n}, F=\operatorname{diag}\left(F_{1}, F_{2}, \ldots, F_{n}\right)$, and $G=\operatorname{diag}\left(G_{1}, G_{2}\right.$, $\left.\ldots, G_{n}\right)$;

(C2) There is a positive number $\gamma>0$ such that

$$
\sup _{l \in N}\left\{\frac{\ln \gamma_{l}}{t_{l}-t_{l-1}}\right\} \leq \gamma<\lambda \text {, }
$$

where $\gamma_{l}=\max _{1 \leq p \leq n}\left\{1, I_{p l}\right\}, l \in N$; then, systems (1a)-(1d) are globally exponentially stable.

Proof. Note that $\bar{\zeta}(\lambda) \geq \zeta(\lambda)$, it follows that

$$
B(t) G \otimes \bar{\zeta}(\lambda) \geq B(t) G \otimes \zeta(\lambda), \quad t \geq 0 .
$$

This shows that $\left(C 1^{\prime \prime}\right)$ ensures $(\mathrm{C} 1)$.

In this part, we consider the case of the periodic solution of systems (1a)-(1d). Let us make the following assumptions:

(A4) For $p, q \in\{1,2, \ldots, n\}, \quad \beta_{p}(t), a_{p q}(t)$, $b_{p q}(t), \tau_{p q}(t)$, and $J_{p}(t)$ are periodic continuous functions and has a common period $\Omega>0$ for all $t \geq 0$.

(A5) For $\mathfrak{\Im}_{l}=\operatorname{diag}\left(I_{1 l}, \ldots, I_{n l}\right)$ and the impulsive moments $\left\{t_{l}\right\}_{l \in N}$, there exists an integer $r>0$ such that

$$
\begin{aligned}
I_{p(l+r)} & =I_{p l}, \\
t_{l+r} & =t_{l}+\Omega .
\end{aligned}
$$

For the existence and the global exponential stability of periodic solutions for periodic systems (1a)-(1d), we have the following results.

Theorem 3. Under assumptions $(A 1)-(A 5)$, suppose that (C1) There exist a vector $\xi=\left(\xi_{1}, \xi_{2}, \ldots, \xi_{n}\right)^{T}>0$ and $a$ constant $\lambda>0$ such that

$$
[\lambda E-D(t)+A(t) F+B(t) G \otimes \zeta(\lambda)] \xi<0, \quad t \geq 0,
$$

where $D(t)=\operatorname{diag}\left(d_{1}(t), d_{2}(t), \ldots, d_{n}(t)\right)$ with $d_{p}(t)$ $=\beta_{p}(t)+\sum_{k=1}^{m}\left(D_{p k} / L_{k}^{2}\right), \quad \zeta(\lambda)=\left(e^{\lambda \tau_{p q}(t)}\right)_{n \times n}, \quad A(t)=$ $\left(a_{p q}^{+}(t)\right)_{n \times n}, B(t)=\left(b_{p q}^{+}(t)\right)_{n \times n}, \quad F=\operatorname{diag}\left(F_{1}, F_{2}, \ldots\right.$, $\left.F_{n}\right)$, and $G=\operatorname{diag}\left(G_{1}, G_{2}, \ldots, G_{n}\right)$;

(C2) There is a positive number $\gamma>0$ such that

$$
\sup _{l \in N}\left\{\frac{\ln \gamma_{l}}{t_{l}-t_{l-1}}\right\} \leq \gamma<\lambda
$$

where $\gamma_{l}=\max _{1 \leq p \leq n}\left\{1, I_{p l}\right\}, l \in N$; then, systems (1a)-(1d) have exactly one globally exponentially stable $\Omega$-periodic solution.

Proof. On the basis of Theorem 1, there exists a integer $K>0$ such that $M e^{-(\lambda-\gamma) K \Omega} \leq(1 / 2)$, and we define a Poincare mapping as follows:

$$
\Gamma: P C(\Xi) \longrightarrow P C(\Xi), \quad \text { by } \Gamma(\omega)=z_{\Omega}(\omega, x),
$$

and it follows that $\Gamma^{K}(\omega)=z_{K \Omega}(\omega, x)$. Setting $t=K \Omega$, we get

$$
\left\|\Gamma^{K}(\omega)-\Gamma^{K}(\omega)\right\| \leq \frac{1}{2}\|\omega-\varpi\| .
$$

Obviously, $\Gamma^{K}$ is a contraction mapping, and then there exists a unique fixed point $\omega^{*} \in P C(\Xi)$ such that

$$
\Gamma^{K}\left(\omega^{*}\right)=\omega^{*} \text {. }
$$

And then, we have

$$
\Gamma^{K}\left(\Gamma\left(\omega^{*}\right)\right)=\Gamma\left(\Gamma^{K}\left(\omega^{*}\right)\right)=\Gamma\left(\omega^{*}\right),
$$

and this implies that $\Gamma\left(\omega^{*}\right) \in P C(\Xi)$ is also a fixed point of $\Gamma^{K}$. So, we have

$$
\Gamma\left(\omega^{*}\right)=\omega^{*}, \quad \text { i.e. } z_{\Omega}\left(\omega^{*}, x\right)=\omega^{*} .
$$


Hence, if $z\left(t, \omega^{*}, x\right)$ is a solution of systems (1a)-(1d) through $\left(0, \omega^{*}\right)$, then $z\left(t+\Omega, \omega^{*}, x\right)$ is also a solution to systems (1a)-(1d). It follows that

$$
z_{t+\Omega}\left(\omega^{*}, x\right)=z_{t}\left(z_{\Omega}\left(\omega^{*}, x\right)\right)=z_{t}\left(\omega^{*}, x\right), \quad t \geq 0,
$$

that is,

$$
z\left(t+\Omega, \omega^{*}, x\right)=z\left(t, \omega^{*}, x\right) .
$$

This demonstrates that $z\left(t, \omega^{*}, x\right)$ has a solution for systems (1a)-(1d) with $\Omega$-period, and all other solutions of systems (1a)-(1d) converge exponentially to it as $t \longrightarrow+\infty$. The proof is completed.

Corollary 5. Under assumptions $(A 1)-(A 5)$,

$\left(C 1^{\prime}\right)$ There exist constants $\xi_{p}>0(p=1,2, \ldots, n)$ and $a$ number $\lambda>0$ such that

$$
\left(\beta_{p}(t)+\sum_{k=1}^{m} \frac{D_{p k}}{L_{k}^{2}}\right)-\sum_{q=1}^{n} \frac{\xi_{q}}{\xi_{p}} a_{p q}^{+}(t) F_{q}-\sum_{q=1}^{n} \frac{\xi_{q}}{\xi_{p}} b_{p q}^{+}(t) e^{\lambda \tau_{p q}(t)} G_{q}>\lambda>0, \quad \text { for all } t \geq 0, p=1,2, \ldots, n \text {. }
$$

(C2) There exists a positive number $\gamma>0$ such that

$$
\sup _{l \in N}\left\{\frac{\ln \gamma_{l}}{t_{l}-t_{l-1}}\right\} \leq \gamma<\lambda,
$$

where $\gamma_{l}=\max _{1 \leq p \leq n}\left\{1, I_{p l}\right\}, l \in N$; then, systems (1a)-(1d) have exactly one globally exponentially stable $\Omega$-periodic solution.
Corollary 6. Under assumptions ( $A 1)$ and ( $A 4)$, if condition $(C 1)$ holds, then systems (2a)-(2c) have exactly one globally exponentially stable $\Omega$-periodic solution.

\section{Illustrative Examples}

In this section, we give two examples to illustrate the conditions required for our theorems.

Example 1. Consider a two-neuron impulsive system with reaction-diffusion terms:

$$
\begin{aligned}
\frac{\partial z_{p}(t, x)}{\partial t}= & \sum_{k=1}^{1} \frac{\partial}{\partial x_{k}}\left(D_{p k} \frac{\partial z_{p}(t, x)}{\partial x_{k}}\right)-\beta_{p}(t) z_{p}(t, x)+\sum_{q=1}^{n} a_{p q}(t) f_{q}\left(z_{q}(t, x)\right) \\
& +\sum_{q=1}^{n} b_{p q}(t) g_{q}\left(z_{q}\left(t-\tau_{p q}(t)\right), x\right), \quad t \geq 0, t \neq t_{l}, x \in \Xi, \\
z_{p}\left(t_{l}^{+}, x\right)= & 1.4 z_{p}\left(t_{l}^{-}, x\right), \quad x \in \Xi, l \in N, \\
z_{p}(t, x)= & 0, \quad t \geq 0, x \in \partial \Xi, \\
z_{p}(s, x)= & \mu_{p}(s, x), \quad s \in[-\tau, 0],
\end{aligned}
$$

for $p=1,2$, where $\Xi=[-1,1], t_{l}=1.5 \pi l$, and

$$
\begin{aligned}
\left(D_{p 1}\right) & =\left(\begin{array}{c}
0.15 \\
0.15
\end{array}\right), \\
\beta(t) & =\left(\begin{array}{cc}
|\sin \pi t| & 0 \\
0 & |\cos 3 t|
\end{array}\right), \\
A(t) & =\left(\begin{array}{cc}
|\sin \pi t| & \cos 4 t \\
\sin t & |\cos 3 t|
\end{array}\right),
\end{aligned}
$$

$$
\begin{aligned}
B(t) & =\left(\begin{array}{cc}
\frac{1}{25}\left(1-e^{-t}\right) & \cos 2 t \\
\sin t & \frac{1-e^{-t}}{30\left(1+e^{-t}\right)}
\end{array}\right), \\
(\tau(t))_{2 \times 2} & =\left(\begin{array}{cc}
|\sin t| & |\sin t| \\
|\cos t| & |\cos t|
\end{array}\right), \\
f_{q}(z) & =g_{q}(z)=\frac{|z+1|-|z-1|}{2} .
\end{aligned}
$$

It is easy to verify that assumptions (A1)-(A3) are satisfied, and we can obtain the following results: 


$$
\begin{aligned}
\zeta(\lambda) & =\left(\begin{array}{cc}
e^{\lambda|\sin t|} & e^{\lambda|\sin t|} \\
e^{\lambda|\cos t|} & e^{\lambda|\cos t|}
\end{array}\right), \\
D(t) & =\left(\begin{array}{cc}
0.15+|\sin \pi t| & 0 \\
0 & 0.15+|\cos 3 t|
\end{array}\right), \\
F & =G=\left(\begin{array}{ll}
1 & 0 \\
0 & 1
\end{array}\right),
\end{aligned}
$$

$\lambda \in[0,1), \gamma_{l}=\max \{1,1.4\}=1.4 \geq 1, \quad \gamma=\sup _{l \in N}\left(\ln \gamma_{l} /\left(t_{l}-\right.\right.$ $\left.\left.t_{l-1}\right)\right)=(\ln 1.4 / 1.5 \pi) \approx 0.0714$.

By solving the following optimization problem:

$$
\text { (OP) } \begin{cases}\max & \lambda \\ \text { s.t. } & (C 1) \text { holds, }\end{cases}
$$

we can obtain that $\lambda \approx 0.1054>0.0714=\gamma$ and $\xi=(233240509,227642892)>0$. Thus, the conditions in Theorem 1 hold, then the solution of systems (69a)-(69d) is globally exponentially stable, and the exponential convergence rate is estimated as $\lambda-\gamma \approx 0.1054-0.0714=0.0340$. The numerical simulation of systems $(69 a)-(69 d)$ is shown in Figure 1.

Example 2. Consider a two-neuron periodic impulsive system: for $p=1,2$, where $\Xi=[-1,1], t_{l}=1.5 \pi l$, and

$$
\begin{aligned}
\left(D_{p 1}\right) & =\left(\begin{array}{c}
0.15 \\
0.15
\end{array}\right), \\
\beta(t) & =\left(\begin{array}{cc}
|\sin t| & 0 \\
0 & |\cos t|
\end{array}\right), \\
A(t) & =\left(\begin{array}{cc}
|\sin t| & \cos t \\
\sin t & |\cos t|
\end{array}\right), \\
B(t) & =\left(\begin{array}{cc}
\frac{1}{20}|\sin t| & \cos t \\
\sin t & \frac{1}{30}|\cos t|
\end{array}\right), \\
(\tau(t))_{2 \times 2} & =\left(\begin{array}{cc}
|\sin t| & |\sin t| \\
|\cos t| & |\cos t|
\end{array}\right), \\
f_{q}(z) & =g_{q}(z)=\frac{|z+1|-|z-1|}{2} .
\end{aligned}
$$

It is easy to verify that assumptions (A1)-(A5) are satisfied, and we can obtain the following results:

$$
\begin{aligned}
\zeta(\lambda) & =\left(\begin{array}{cc}
e^{\lambda|\sin t|} & e^{\lambda|\sin t|} \\
e^{\lambda|\cos t|} & e^{\lambda|\cos t|}
\end{array}\right), \\
D(t) & =\left(\begin{array}{cc}
0.15+|\sin t| & 0 \\
0 & 0.15+|\cos t|
\end{array}\right), \\
F & =G=\left(\begin{array}{ll}
1 & 0 \\
0 & 1
\end{array}\right),
\end{aligned}
$$

$\lambda \in[0,1), \gamma_{l}=\max \{1,1.5\}=1.5 \geq 1, \gamma=\sup _{l \in N}\left(\ln \gamma_{l} /\left(t_{l}-\right.\right.$ $\left.\left.t_{l-} 1\right)\right)=(\ln 1.5 / 1.5 \pi) \approx 0.0860$.

By solving the following optimization problem:

$$
\text { (OP) } \begin{cases}\max & \lambda \\ \text { s.t. } & (\mathrm{C} 1) \text { holds, }\end{cases}
$$

we can obtain that $\lambda \approx 0.0950>0.0860=\gamma$ and $\xi=(106069006,130884350)>0$. Thus, the conditions in Theorem 3 hold, then systems (73a)-(73d) have exactly one globally exponentially stable $2 \pi$-periodic solution, and the exponential convergence rate is estimated as $\lambda-\gamma \approx$ $0.0950-0.0860=0.0090$. The numerical simulation of systems $(73 \mathrm{a})-(73 \mathrm{~d})$ is shown in Figure 2. 

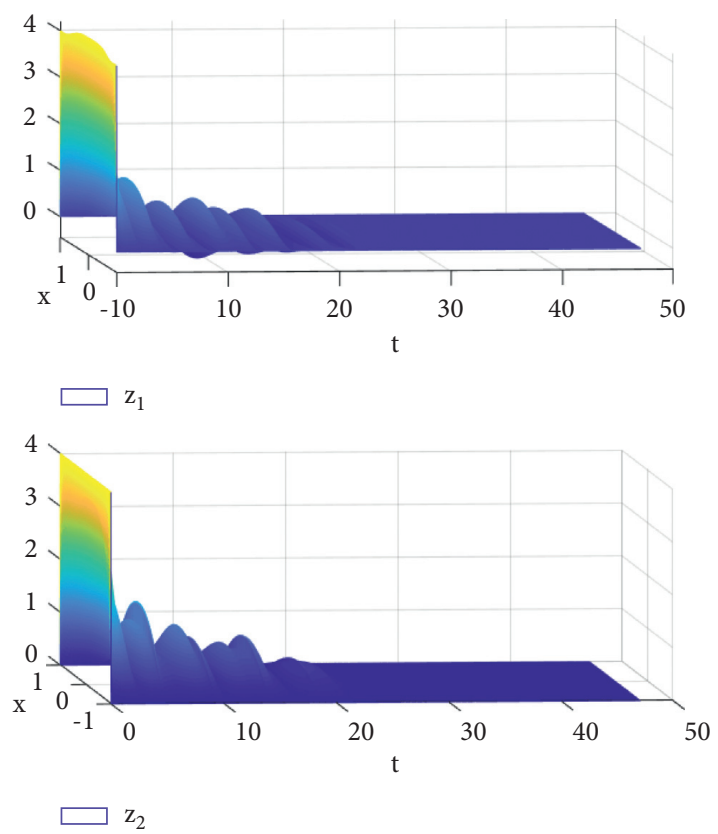

Figure 1: Computer simulation of the neuronal states $z_{1}(t, x)$ and $z_{2}(t, x)$.
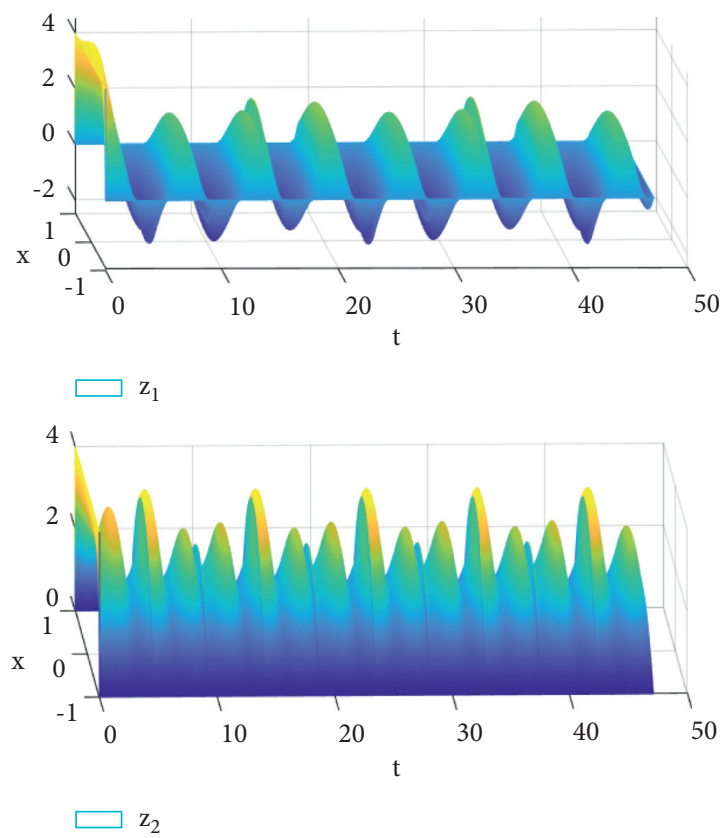

FIGURE 2: Computer simulation of the neuronal states $z_{1}(t, x)$ and $z_{2}(t, x)$.

\section{Conclusion}

We have formulated and investigated a class of new neural network models which assembles nonautonomous neural networks, reaction-diffusion cellular neural networks with time-varying delays, impulses, and the Dirichlet boundary conditions. Several new sufficient conditions have been obtained to ensure the global exponential stability and periodicity of systems (1a)-(1d), and these criteria are shown in simple algebraic inequality forms. In particular, an optimization method is proposed in order to estimate the exponential convergence rate of systems (1a)-(1d), and this method depends on the diffusion coefficient, the Dirichlet boundary conditions, the delays, system parameters, and impulses. Comparing with the method of Lyapunov functional in many previous publications, our method is simpler and more effective for stability and periodicity analysis of nonautonomous impulsive neural networks with timevarying delays and reaction-diffusion terms. Two examples have shown that our results improve and generalize previously known criteria. In near future, we will continue to study global exponential stability and periodicity of nonautonomous impulsive neural networks with distributed delays or leakage delays under the Dirichlet boundary conditions.

\section{Data Availability}

The data used to support the findings of this study are available from the corresponding author upon request.

\section{Conflicts of Interest}

The authors declare that they have no conflicts of interest.

\section{Acknowledgments}

This work was supported by the National Natural Science Foundation of China under Grant 61573010.

\section{References}

[1] L. O. Chua and L. Yang, "Cellular neural networks: theory," IEEE Transactions on Circuits and Systems, vol. 35, no. 10, pp. 1257-1272, 1988.

[2] W.-H. Chen and W. X. Zheng, "Improved delay-dependent asymptotic stability criteria for delayed neural networks," IEEE Transactions on Neural Networks, vol. 19, no. 12, pp. 2154-2161, 2008.

[3] C.-C. Hua, X. Yang, J. Yan, and X.-P. Guan, "New exponential stability criteria for neural networks with time-varying delay," IEEE Transactions on Circuits and Systems II: Express Briefs, vol. 58, no. 12, pp. 931-935, 2011.

[4] X. Liu, X. Liu, M. Tang, and F. Wang, "Improved exponential stability criterion for neural networks with time-varying delay," Neurocomputing, vol. 234, pp. 154-163, 2017.

[5] M.-D. Ji, Y. He, C.-K. Zhang, and M. Wu, "Novel stability criteria for recurrent neural networks with time-varying delay," Neurocomputing, vol. 138, pp. 383-391, 2014.

[6] L. Hua, H. Zhu, K. Shi, S. Zhong, Y. Tang, and Y. Liu, "Novel finite-time reliable control design for memristor-based inertial neural networks with mixed time-varying delays," IEEE Transactions on Circuits and Systems I: Regular Papers, vol. 68, no. 4, pp. 1599-1609, 2021.

[7] K. Shi, J. Wang, Y. Tang, and S. Zhong, "Reliable asynchronous sampled-data filtering of T-S fuzzy uncertain delayed neural networks with stochastic switched topologies," Fuzzy Sets and Systems, vol. 381, pp. 1-25, 2020.

[8] L. O. Chua, M. Hasler, G. S. Moschytz, and J. Neirynck, "Autonomous cellular neural networks: a unified paradigm for pattern formation and active wave propagation," IEEE 
Transactions on Circuits and Systems I: Fundamental Theory and Applications, vol. 42, no. 10, pp. 559-577, 1995.

[9] J. Liang and J. Cao, "Global exponential stability of reactiondiffusion recurrent neural networks with time-varying delays," Physics Letters A, vol. 314, no. 5-6, pp. 434-442, 2003.

[10] L. Wang and Y. Gao, "Global exponential robust stability of reaction-diffusion interval neural networks with time-varying delays," Physics Letters A, vol. 350, no. 5-6, pp. 342-348, 2006.

[11] T. Huang, "Exponential stability of delayed fuzzy cellular neural networks with diffusion," Chaos, Solitons \& Fractals, vol. 31, no. 3, pp. 658-664, 2007.

[12] Z. Zhang, Y. Yang, and Y. Huang, "Global exponential stability of interval general BAM neural networks with reactiondiffusion terms and multiple time-varying delays," Neural Networks, vol. 24, no. 5, pp. 457-465, 2011.

[13] Z. Wang and H. Zhang, "Global asymptotic stability of reaction-diffusion Cohen-Grossberg neural networks with continuously distributed delays," IEEE Transactions on Neural Networks, vol. 21, no. 1, pp. 39-49, 2010.

[14] J. G. Lu, "Robust global exponential stability for interval reaction-diffusion hopfield neural networks with distributed delays," IEEE Transactions on Circuits and Systems II: Express Briefs, vol. 54, no. 12, pp. 1115-1119, 2007.

[15] J. G. Lu, "Global exponential stability and periodicity of reaction-diffusion delayed recurrent neural networks with Dirichlet boundary conditions," Chaos, Solitons \& Fractals, vol. 35, no. 1, pp. 116-125, 2008.

[16] X. Lu, W.-H. Chen, Z. Ruan, and T. Huang, "A new method for global stability analysis of delayed reaction-diffusion neural networks," Neurocomputing, vol. 317, pp. 127-136, 2018.

[17] J. Pan and S. Zhong, "Novel criteria on global robust exponential stability to a class of reaction-diffusion neural networks with delays," Discrete Dynamics in Nature and Society, vol. 2009, Article ID 291594, , 2009.

[18] R. Rao, "Delay-dependent exponential stability for nonlinear reaction-diffusion uncertain Cohen-Grossberg neural networks with partially known transition rates via HardyPoincaré inequality," Chinese Annals of Mathematics, Series B, vol. 35, no. 4, pp. 575-598, 2014.

[19] H. Jiang and J. Cao, "Global exponential stability of periodic neural networks with time-varying delays," Neurocomputing, vol. 70, no. 1-3, pp. 343-350, 2006.

[20] C. Xu and Q. Zhang, "On antiperiodic solutions for CohenGrossberg shunting inhibitory neural networks with timevarying delays and impulses," Neural Computation, vol. 26, no. 10, pp. 2328-2349, 2014.

[21] C. Xu, Q. Zhang, and Y. Wu, "Existence and stability of pseudo almost periodic solutions for shunting inhibitory cellular neural networks with neutral type delays and timevarying leakage delays," Network: Computation in Neural Systems, vol. 25, no. 4, pp. 168-192, 2014.

[22] H. Zhang and J. Shao, "Almost periodic solutions for cellular neural networks with time-varying delays in leakage terms," Applied Mathematics and Computation, vol. 219, no. 24, pp. 11471-11482, 2013.

[23] S. Long and D. Xu, "Global exponential stability of nonautonomous cellular neural networks with impulses and timevarying delays," Communications in Nonlinear Science and Numerical Simulation, vol. 18, no. 6, pp. 1463-1472, 2013.

[24] C. Xu, M. Liao, P. Li, Z. Liu, and S. Yuan, "New results on pseudo almost periodic solutions of quaternion-valued fuzzy cellular neural networks with delays," Fuzzy Sets and Systems, vol. 411, pp. 25-47, 2021.
[25] X. Lou and B. Cui, "Boundedness and exponential stability for nonautonomous RCNNs with distributed delays," Computers \& Mathematics with Applications, vol. 54, no. 4, pp. 589-598, 2007.

[26] C. Xu, P. Li, and Y. Pang, "Exponential stability of almost periodic solutions for memristor-based neural networks with distributed leakage delays," Neural Computation, vol. 28, no. 12, pp. 2726-2756, 2016.

[27] S. Long, H. Li, and Y. Zhang, "Dynamic behavior of nonautonomous cellular neural networks with time-varying delays," Neurocomputing, vol. 168, pp. 846-852, 2015.

[28] J. J. Oliveira, "Global exponential stability of nonautonomous neural network models with unbounded delays," Neural Networks, vol. 96, pp. 71-79, 2017.

[29] K. Li, "Stability analysis for impulsive Cohen-Grossberg neural networks with time-varying delays and distributed delays," Nonlinear Analysis: Real World Applications, vol. 10, no. 5, pp. 2784-2798, 2009.

[30] H. Li, C. Li, and T. Huang, "Periodicity and stability for variable-time impulsive neural networks," Neural Networks, vol. 94, pp. 24-33, 2017.

[31] F. Meng, K. Li, Q. Song, Y. Liu, and F. E. Alsaadi, "Periodicity of Cohen-Grossberg-type fuzzy neural networks with impulses and time-varying delays," Neurocomputing, vol. 325, pp. 254-259, 2019.

[32] B. Li and Q. Song, "Some new results on periodic solution of Cohen-Grossberg neural network with impulses," Neurocomputing, vol. 177, pp. 401-408, 2016.

[33] F. Miaadi and X. Li, "Impulse-dependent settling-time for finite time stabilization of uncertain impulsive static neural networks with leakage delay and distributed delays," Mathematics and Computers in Simulation, vol. 182, pp. 259-276, 2021.

[34] A. Berman and R. J. Plemmons, Nonnegative Matrices in the Mathematical Science, Academic Press, Cambridge, MA, USA, 1979.

[35] J. Wang and J. G. Lu, "Global exponential stability of fuzzy cellular neural networks with delays and reaction-diffusion terms," Chaos, Solitons \& Fractals, vol. 38, no. 3, pp. 878-885, 2008.

[36] W. Zhao, "Dynamics of Cohen-Grossberg neural network with variable coefficients and time-varying delays," Nonlinear Analysis: Real World Applications, vol. 9, no. 3, pp. 1024-1037, 2008.

[37] Z. Yang and D. Xu, "Global dynamics for non-autonomous reaction-diffusion neural networks with time-varying delays," Theoretical Computer Science, vol. 403, no. 1, pp. 3-10, 2008.

[38] J. Li, F. Zhang, and J. Yan, "Global exponential stability of nonautonomous neural networks with time-varying delays and reaction-diffusion terms," Journal of Computational and Applied Mathematics, vol. 233, no. 2, pp. 241-247, 2009. 